A) Check for updates

Cite this: DOI: $10.1039 / \mathrm{c7fo00859g}$

\section{The protective effect of acerola (Malpighia emarginata) against oxidative damage in human dermal fibroblasts through the improvement of antioxidant enzyme activity and mitochondrial functionality}

\author{
José M. Alvarez-Suarez, (iD ${ }^{\mathrm{a}, \mathrm{b}}$ Francesca Giampieri, ${ }^{\star b}$ Massimiliano Gasparrini, ${ }^{\mathrm{b}}$ \\ Luca Mazzoni, ${ }^{\mathrm{C}}$ Celestino Santos-Buelga, (D) d Ana M. González-Paramás, ${ }^{d}$ \\ Tamara Y. Forbes-Hernández, ${ }^{b}$ e Sadia Afrin, ${ }^{b}$ Timothy Páez-Watson, ${ }^{f}{ }^{\prime}$ José L. Quiles ${ }^{g}$ \\ and Maurizio Battino (iD) *b,e
}

\begin{abstract}
Acerola fruits (Malpighia emarginata DC.) from the central region of Cuba were analyzed to determine their chemical composition and protective capacity against oxidative damage using an in vitro human dermal fibroblast (HDFa) model. The chemical composition analyses showed a high content of vitamin C, total polyphenols, $\beta$-carotene and folates in the acerola fruit. From the HPLC-DAD/ESI-MS ${ }^{n}$ analyses, two anthocyanins (cyanidin 3-O-rhamnoside and pelargonidin 3-O-rhamnoside), three hydroxycinnamoyl derivatives (caffeoyl hexoside, dihydrocaffeoylquinic acid and coumaroyl hexoside) and fifteen flavonols (mostly glycosylated forms of quercetin and kaempferol) were detected. HDFa were pre-incubated with an acerola crude extract (ACExt) and subsequently subjected to oxidative stress induced by AAPH. Apoptosis, intracellular ROS and the biomarkers of lipid and protein oxidation significantly increased after inducing stress, while the activities of the antioxidant enzyme catalase and superoxide dismutase and mitochondrial functionality were markedly affected. However, ACExt was able to protect against oxidative damage through decreasing apoptosis, intracellular ROS levels and lipid and protein damage, besides improving antioxidant enzyme activities and mitochondrial functionality. The obtained results support acerola fruits as relevant sources of functional compounds with promising effects on human health.
\end{abstract}

Received 12th June 2017, Accepted 29th July 2017 DOI: $10.1039 / \mathrm{c} 7 f \circ 00859 \mathrm{~g}$ rsc.li/food-function
In this sense, it has been recognized that polyphenols and other phytochemicals, such as vitamin $\mathrm{C}$ and carotenoids, possess important biological properties related to their antioxidant capacity and the ability to activate certain molecular pathways related to the antioxidant response. ${ }^{1,3}$ Based on these approaches the current search for dietary sources of bioactive compounds that may offer protection against oxidative stress-related-diseases is a continuous mission for modern science.

Acerola fruits are produced by the acerola tree (Malpighia emarginata DC.), a tropical fruit-bearing shrub or small tree in the Malpighiaceae family that is native to the Caribbean islands, Central and Northern South America, and the Amazonian region. ${ }^{4}$ The acerola fruit is well known for its high content of vitamin $\mathrm{C}$, which makes it a natural and excellent source of this compound. Moreover, since it is a red fruit, it is expected to contain other important functional compounds, such as those of the family of color-related polyphenols, like anthocyanins, and therefore its biological properties could also be related to the beneficial effects associated with this 
type of compounds. ${ }^{1}$ Since to date no studies of this type have been reported on acerola fruit, our research sought to determine the protective effects of a crude extract of acerola against the cytotoxic damage mediated by induced oxidative stress, through the study of the markers of oxidative stress and cellular damage. These include intracellular ROS production, cellular apoptosis, lipid and protein oxidation, antioxidant enzyme activities and the evaluation of the mitochondrial respiration rate using an in vitro human dermal fibroblast model.

\section{Materials and methods}

\section{Fruit preparation and analysis}

Ripe acerola fruits (Malpighia emarginata DC.) were harvested from plants grown in the provinces of Sancti Spiritus and Cienfuegos, in the central region of Cuba. The specimen (Malpighia emarginata DC.: Malpighiaceae) was identified by specialists of the Fruit Research Institute of Havana, Cuba, using the reference samples deposited in the herbarium of the Institute. Moreover a voucher from the collected samples was also deposited in the herbarium of the Institute. Within $2 \mathrm{~h}$ after harvest, the whole fruits were stored at $-20{ }^{\circ} \mathrm{C}$ until future sample preparation for analysis (not more than 3 months). Prior to the analysis, the edible parts were freezedried, ground to a fine powder, stored at $-20{ }^{\circ} \mathrm{C}$ and then extracted using a hydroalcoholic solution (methanol: water, $80: 20 \mathrm{v} / \mathrm{v}$ ) as previously reported. ${ }^{5}$

Hydroalcoholic extracts were used for spectrophotometric analysis of total phenolic content (TPC), ${ }^{6}$ total flavonoid content $(\mathrm{TFC})^{7}$ and total anthocyanin content (ACYs) ${ }^{8}$ and the results were expressed as milligrams of gallic acid equivalents (GAE) per gram of fresh weight of fruit (FW) (mg GAE per $\mathrm{g}$ FW), milligrams of catechin equivalents (CatEq) per gram of FW (mg CatEq per $\mathrm{g}$ FW) and milligrams of Pg-3-gluc equivalents (PgEq) per gram of FW (mg PgEq per $\mathrm{g} F W$ ), respectively.

\section{HPLC-DAD determination of vitamin C}

Ascorbic acid was determined as previously reported by our group. ${ }^{9}$ Briefly, $2 \mathrm{~mL}$ of ice-cold water with $5 \%$ metaphosphoric acid and $1 \mathrm{mM}$ DTPA were added to $0.5 \mathrm{~g}$ of freeze-dried fruit powder, sonicated for $3 \mathrm{~min}$ and then centrifuged at 2500 rpm for $10 \mathrm{~min}$, filtered, and immediately analysed on an HPLC system. Analyses were performed using a HPLC system (Shimadzu Corp., Kyoto, Japan) consisting of a Waters 600 controller, and a Waters 996 photodiode array (PDA) detector set at absorbances of 262 and $244 \mathrm{~nm}$. A YMC Pack Pro column $(150 \times 4.6 \mathrm{~mm})$ was used as the stationary phase and elution was performed with $50 \mathrm{mM} \mathrm{KH_{2 }} \mathrm{PO}_{4}(\mathrm{pH} 4.5)$ at a flow rate of $0.8 \mathrm{~mL} \mathrm{~min}^{-1}$ for $15 \mathrm{~min}$ and the results were expressed as $\mathrm{mg}$ per $100 \mathrm{~g}$ FW.

\section{HPLC-DAD analysis of $\beta$-carotene}

For $\beta$-carotene analysis, extraction was carried out as previously reported. ${ }^{10}$ Five grams of freeze-dried fruit powder were added to $30 \mathrm{~mL}$ of acetone, sonicated for $15 \mathrm{~min}$, stirred for $1 \mathrm{~h}$ in the dark at room temperature, and then centrifuged at 9000 $\mathrm{rpm}$ at $4{ }^{\circ} \mathrm{C}$ for $15 \mathrm{~min}$. The extracted solution was then subjected to saponification using a methanolic solution of $\mathrm{KOH}$ $(10 \%)$ overnight at room temperature. This substance was then extracted with petroleum ether and the organic layer was dried under vacuum in a rotary evaporator. The dried residue was dissolved in hexane and filtered through a $0.45 \mathrm{~mm}$ membrane before HPLC analysis. The HPLC-DAD system (Shimadzu Corp., Kyoto, Japan) consisted of a Waters 600 controller and a Waters 996 photodiode array (PDA) detector set at an absorbance of $450 \mathrm{~nm}$ and equipped with a Supelcosil ${ }^{\mathrm{TM}}$ LC-18 $(150 \times 4.6 \mathrm{~mm})$ as the stationary phase. Elution was performed with acetonitrile/methanol/ethyl acetate $(88: 10: 2 \mathrm{v} / \mathrm{v})$ in an isocratic gradient at a flow rate of $1 \mathrm{~mL} \mathrm{~min}^{-1}$ and the results were expressed as $\mathrm{mg} \beta$-carotene per $100 \mathrm{~g}$ FW.

\section{RP-HPLC determination of folic acid}

Folate extraction was performed as previously reported by Shohag et $a l .{ }^{11}$ Briefly, $5 \mathrm{~g}$ of frozen acerola fruit were homogenized in a extraction buffer $(0.1 \mathrm{M}$ phosphate buffer containing $1.0 \%$ of $\mathrm{L}(+)$-ascorbic acid $(\mathrm{w} / \mathrm{v})$ and $0.1 \%$ 2,3-dimercapto-1-propanol (v/v) at $\mathrm{pH}$ 6.5) using an Ultraturrax T25 homogenizer (Janke \& Kunkel, IKA Labortechnik). The extracted fraction was placed in a water bath at $100{ }^{\circ} \mathrm{C}$ for $10 \mathrm{~min}$, rapidly cooled on ice and centrifuged at $27000 \mathrm{~g}$ for $20 \mathrm{~min}$ at $4{ }^{\circ} \mathrm{C}$. The supernatants were completed to a final volume of $25 \mathrm{ml}$ with the extraction buffer and the deconjugation of polyglutamylated folates was induced by adding $175 \mu \mathrm{l}$ of folate conjugase from rat serum to $5 \mathrm{ml}$ of the extract. The reaction mixture was then incubated in a shaking oven at $37^{\circ} \mathrm{C}$ for $2 \mathrm{~h}$. Folate conjugase was obtained from rat serum as previously reported by Shohag et al. ${ }^{11}$ After incubation, the samples were treated for $5 \mathrm{~min}$ at $100{ }^{\circ} \mathrm{C}$ and centrifuged at $27000 \mathrm{~g}$ for $20 \mathrm{~min}$ at $4^{\circ} \mathrm{C}$ for $20 \mathrm{~min}$. The supernatant was filtered through a Minisart filter of $45 \mu \mathrm{m}$ (PBI International, Milan, Italy) and then purified through solid-phase extraction (SPE) on strong anion-exchange (SAX) Isolute cartridges (3 mL per $500 \mathrm{mg}$ of quaternary amine $\mathrm{N}^{+}$, counter ion $\mathrm{Cl}^{-}$, Supelco, Bellefonte, PA). Aliquots $(2.5 \mathrm{~mL})$ of the sample extracts were passed through cartridges and the elution of retained folates was performed with $0.1 \mathrm{M}$ sodium acetate containing $10 \%$ sodium chloride (w/v), 1\% L(+)-ascorbic acid (w/v), and $0.1 \%$ 2,3-dimercapto-1-propanol (v/v).

Chromatographic separation was performed using an HPLC system (Jasco PU-2089 Plus), which consisted of a gradient binary pump, a UV detector (Jasco UV-2070 Plus), a fluorescence detector (FLD) (Jasco FP-2020 Plus), and a computer running ChromNAV software. The stationary phase consisted of a Mediterranea Sea18 column $(250 \times 4.6 \mathrm{~mm})$ and the mobile phase was a binary gradient mixture of $30 \mathrm{mM}$ potassium phosphate buffer at $\mathrm{pH} 2.3$ (A) and acetonitrile (B). The gradient program was as follows: $\mathrm{B}$ was kept in isocratic mode for $5 \mathrm{~min}(6 \% \mathrm{v} / \mathrm{v})$, raised linearly to $25 \%$ within $20 \mathrm{~min}$ and kept constant for $2 \mathrm{~min}$; then $\mathrm{B}$ was decreased linearly to $6 \%$ for $1 \mathrm{~min}$ and was applied for $14 \mathrm{~min}$ to re-equilibrate the column. The flow rate was $0.4 \mathrm{~mL} \mathrm{~min}^{-1}$ and the folates were 
detected and quantized with a FLD detector set at excitation/ emission of 290/360 $\mathrm{nm}$ for reduced folates and 360/460 nm for 10-formylfolic acid (10-HCO-folic acid) and a UV detector set at $290 \mathrm{~nm}$. The results are expressed as $\mu \mathrm{g}$ of each folate per 100 grams of fresh weight of acerola.

\section{HPLC-DAD/ESI-MS ${ }^{n}$ characterization of polyphenols isolated from acerola fruit}

HPLC analyses were carried out in a Hewlett-Packard 1200 chromatograph (Agilent Technologies, Waldbronn, Germany) equipped with a binary pump and a diode array detector (DAD) coupled to an HP Chem Station (rev. A.05.04) dataprocessing station. The HPLC system was connected via the DAD cell outlet to an API 3200 Qtrap (Applied Biosystems, Darmstadt, Germany) mass spectrometer (MS) consisting of an ESI source and a triple quadrupole-ion trap mass analyzer, which was controlled by the Analyst 5.1 software. Compounds were identified by their retention time, UV-vis spectra and mass spectra, and comparison with our data library and standards when available.

Analysis of anthocyanins. An AQUA® (Phenomenex) reverse phase C18 column $(5 \mu \mathrm{m}, 150 \mathrm{~mm} \times 4.6 \mathrm{~mm})$ thermostated at $35{ }^{\circ} \mathrm{C}$ was used. The solvents were: (A) $0.1 \%$ trifluoroacetic acid and (B) acetonitrile. The elution gradient established was: isocratic $10 \%$ B for $3 \mathrm{~min}, 10-15 \%$ B in $12 \mathrm{~min}$, isocratic $15 \%$ B for $5 \mathrm{~min}, 15-18 \%$ B over $5 \mathrm{~min}, 18-30 \%$ B over $20 \mathrm{~min}$, $30-35 \%$ B over $5 \mathrm{~min}$, and re-equilibration of the column to initial solvent conditions. The flow rate used was $0.5 \mathrm{~mL}$ $\min ^{-1}$. Double online detection was carried out in the DAD using 280 and $520 \mathrm{~nm}$ as preferred wavelengths, and in the MS operated in the positive ion mode. Spectra were recorded between $\mathrm{m} / \mathrm{z} 100$ and $\mathrm{m} / \mathrm{z}$ 1500. Zero grade air served as the nebulizer gas (40 psi) and as turbo gas $\left(600^{\circ} \mathrm{C}\right)$ for solvent drying (50 psi). Nitrogen served as the curtain (100 psi) and collision gas (high). Both quadrupoles were set at unit resolution and the MS detector was programmed to perform a series of two consecutive analyses, a full scan of high sensitivity (Enhanced MS, EMS) and an Enhanced Product Ion analysis (EPI) to obtain the fragmentation pattern of the parent ion. The EMS mode parameters were the following: ion spray voltage $5000 \mathrm{~V}$, declustering potential (DP) $41 \mathrm{~V}$, entrance potential (EP) $7.5 \mathrm{~V}$ and collision energy (CE) $10 \mathrm{~V}$. EPI mode was applied using the following settings: DP $41 \mathrm{~V}, \mathrm{EP} 7.5 \mathrm{~V}, \mathrm{CE}$ $10 \mathrm{~V}$ and collision energy spread (CES) $0 \mathrm{~V}$.

Analysis of flavonols and other phenolic derivatives. An Agilent Poroshell 120 EC-C18 column $(2.7 \mu \mathrm{m}, 150 \mathrm{~mm}$ x $4.6 \mathrm{~mm}$ ) thermostated at $35^{\circ} \mathrm{C}$ was used. The solvents were: (A) $0.1 \%$ formic acid and (B) acetonitrile. The elution gradient established was isocratic 15\% B for $5 \mathrm{~min}, 15-20 \% \mathrm{~B}$ over $5 \mathrm{~min}, 20-35 \%$ B over $10 \mathrm{~min}, 35-50 \%$ B over $10 \mathrm{~min}, 50-60 \%$ B over $5 \mathrm{~min}$, isocratic $60 \% \mathrm{~B}$ for $5 \mathrm{~min}$ and re-equilibration of the column to initial solvent conditions. The flow rate was $0.5 \mathrm{~mL} \mathrm{~min}^{-1}$. Double online detection was carried out in the $\mathrm{DAD}$ at 280, 330 and $370 \mathrm{~nm}$ as preferred wavelengths and in the MS operated in the negative ion mode. Spectra were recorded between $\mathrm{m} / \mathrm{z} 100$ and $\mathrm{m} / \mathrm{z}$ 1500. Zero grade air served as the nebulizer gas (30 psi) and as turbo gas $\left(400{ }^{\circ} \mathrm{C}\right)$ for solvent drying (40 psi). Nitrogen served as the curtain (20 psi) and collision gas (medium). Both quadrupoles were set at unit resolution and EMS and EPI analyses were also performed. The EMS parameters were: ion spray voltage $4500 \mathrm{~V}, \mathrm{DP}-50 \mathrm{~V}$, EP $-6 \mathrm{~V}, \mathrm{CE}-10 \mathrm{~V}$ and cell exit potential (CXP) $-3 \mathrm{~V}$, whereas EPI settings were: DP $-50 \mathrm{~V}, \mathrm{EP}-6 \mathrm{~V}, \mathrm{CE}-25 \mathrm{~V}$ and CES $0 \mathrm{~V}$.

\section{Total antioxidant capacity (TAC)}

The total antioxidant capacity (TAC) of hydroalcoholic extracts was determined using the Trolox Equivalent Antioxidant Capacity assay (TEAC), ${ }^{12}$ the Ferric Reducing Antioxidant Power (FRAP) assay ${ }^{13}$ and the 2,2-diphenyl-1-picrylhydrazyl free radical method (DPPH); ${ }^{14}$ the results were expressed as $\mu \mathrm{mol}$ of Trolox equivalents (TEq) per gram of FW ( $\mu$ mol TEq per $g$ FW).

\section{Culture of HuDe cell line and cell treatment}

Human Dermal Fibroblasts, adult (HDFa), were purchased from American Type Culture Collection (ATCC® PCS-201$012^{\mathrm{TM}}$ ) and cultured in $25 \mathrm{~cm}^{2}$ flasks in Eagle's Minimum Essential Medium (EMEM) supplemented with $10 \%$ fetal bovine serum, $2 \mathrm{mM}$ glutamine, and 1\% penicillin-streptomycin antibiotics $\left(100 \mathrm{IU} \mathrm{mL}^{-1}\right.$ penicillin and $100 \mu \mathrm{g} \mathrm{mL} \mathrm{m}^{-1}$ streptomycin) in a humidified atmosphere with $5 \% \mathrm{CO}_{2}$ at $37{ }^{\circ} \mathrm{C}$ and the medium was changed every 2-3 days. Acerola hydroalcoholic extract was dried under vacuum to eliminate total methanol and the resulting crude extract (ACExt) was resuspended in EMEM to achieve the final concentration of $80 \mu \mathrm{g} \mathrm{mL} \mathrm{m}^{-1}$. The stressor 2,2'-azobis(2-amidinopropane)dihydrochloride (AAPH) (10 mM) was used as an inductor of oxidative damage in HDFa. The cells were treated with (i) EMEM only (Ctrl), (ii) ACExt for $24 \mathrm{~h}$ (ACExt), (iii) AAPH (10 mM) for $24 \mathrm{~h}$ (AAPH) or (iv) ACExt for $24 \mathrm{~h}$ and then with AAPH (10 $\mathrm{mM})$ for $24 \mathrm{~h}$ (ACExt + AAPH). The combination of dose/ time of ACExt and AAPH treatments was established according to the MTT viability assay for cytotoxicity studies (data not shown).

\section{Tali ${ }^{\circledR}$ apoptosis assay}

Apoptosis was determined using the TALI ${ }^{\circledR}$ Apoptosis Kit (Invitrogen $^{\mathrm{TM}}$, Life Technologies, Milan, Italy) according to the manufacturer's instructions and as previously reported by our group. ${ }^{15}$ Cells were seeded in a 6-well plate $\left(1.5 \times 10^{5}\right)$, allowed to adhere for $\sim 18 \mathrm{~h}$ and then treated (i, ii, iii, iv). After treatments, the medium was removed by centrifugation and the cells were resuspended in $1 \times$ annexin binding buffer $(\mathrm{ABB})$. Five $\mu \mathrm{L}$ of annexin V Alexa Fluor ${ }^{\circledR} 488$ was added to each $100 \mu \mathrm{L}$ of sample, mixed well and incubated at room temperature in the dark for 20 minutes. After incubation the cells were centrifuged and resuspended in $100 \mu \mathrm{L}$ of $5 \times \mathrm{ABB}$, and then $1 \mu \mathrm{L}$ of Tali ${ }^{\circledR}$ propidium iodide was added to $100 \mu \mathrm{L}$ of the sample, mixed well and incubated at room temperature in the dark for 5 minutes. The cells were analysed using the TALI ${ }^{\circledR}$ Image-Based Cytometer (Invitrogen ${ }^{\mathrm{TM}}$, Life Technologies, Milan, Italy) collecting 20-field per sample and the percentage 
of apoptotic nuclei, dead and live cells was determined based on the respective fluorescence histogram compared with the control (i). The results were expressed as fold increase compared with the control.

\section{TALI® ROS assay}

Intracellular ROS production was determined using the probe CellROX ${ }^{\circledR}$ Orange reagent (Invitrogen ${ }^{\mathrm{TM}}$, Life Technologies, Milan, Italy) according to the manufacturer's instructions and as previously reported by our group. ${ }^{15,16}$ Cells were seeded in a 6-well plate $\left(1.5 \times 10^{5}\right)$, allowed to adhere for $\sim 18 \mathrm{~h}$ and then treated (i, ii, iii, iv). After treatments the medium was removed by centrifugation and the CellROX® Orange reagent was added to $1 \mathrm{~mL}$ of complete medium at a $1: 500(\mathrm{v} / \mathrm{v})$ dilution. The samples were incubated at $37{ }^{\circ} \mathrm{C}$ for $30 \mathrm{~min}$, and then centrifuged at $320 g$; the medium was removed and the cells were resuspended in phosphate-buffered saline solution. The cells were analysed using the TALI ${ }^{\circledR}$ Image-Based Cytometer (Invitrogen $^{\mathrm{TM}}$, Life Technologies, Milan, Italy) collecting 20 -field per sample. Control cells were used to set the baseline levels of ROS and the results were expressed as fold increase compared with the control.

\section{Antioxidant enzyme activities and biomarkers of oxidative stress}

Antioxidant enzyme activities were determined spectrophotometrically by measuring the catalase activity (CAT) through $\mathrm{H}_{2} \mathrm{O}_{2}$ decomposition at $240 \mathrm{~nm}$ as previously reported by Aeby, ${ }^{17}$ while superoxide dismutase activity (SOD) was determined on the basis of the inhibition of nitroblue tetrazolium reduction with further measurement at $540 \mathrm{~nm} ;{ }^{18}$ the results were expressed as U per mg prot per min for both assays. Protein carbonyl levels were analyzed using the DNPH method ${ }^{19}$ and the results were expressed as nmol $\mathrm{mg}^{-1}$ of protein. Lipid peroxidation was determined in parallel using the thiobarbituric acidreactive substances assay (TBARS) ${ }^{20}$ and hydroperoxide levels; ${ }^{21}$ the results were expressed as $\mu \mathrm{M}$ for both assays.

\section{Evaluation of the mitochondrial respiration rate}

The oxygen consumption rate (OCR), as an indicator of mitochondrial functionality, was determined using an XF-24 Extracellular Flux Analyzer (Seahorse Bioscience, Billerica, MA, USA) following the experimental conditions previously described by our group for this cell line. ${ }^{15}$ Before the experiment, cells were seeded in the XF-24 plate for 16 hours and then treated according to the previously described protocol (i, ii, iii, iv). Once the different treatments were completed, the medium was replaced with $450 \mu \mathrm{L}$ per well of XF-24 running media (serum-free medium and supplemented with $25 \mathrm{mM}$ of glucose, $2 \mathrm{mM}$ glutamine and $1 \mathrm{mM}$ sodium pyruvate) and incubated at $37^{\circ} \mathrm{C}$ for $20 \mathrm{~min}$ in the absence of $\mathrm{CO}_{2}$ using the XF Prep Station incubator (Seahorse Bioscience). After preincubation, the OCR was measured in the XF-24 Extracellular Flux Analyzer from the baseline OCR determination and subsequent sequential injections of four compounds that affect the cellular bioenergetic processes, as follows: $55 \mu \mathrm{L}$ of oligomycin $\left(2.5 \mu \mathrm{g} \mathrm{mL} \mathrm{mL}^{-1}\right)$ in port $\mathrm{A}, 61 \mu \mathrm{L}$ of 2,4-dinitrophenol
$(1 \mathrm{mM})$ in port $\mathrm{B}$ and $68 \mu \mathrm{L}$ of antimycin/rotenone $(10 \mu \mathrm{M} /$ $1 \mu \mathrm{M})$ in port $\mathrm{C}$. The samples were analysed in triplicate using five wells per treatment and the results were expressed as pmol of $\mathrm{O}_{2}$ consumed per minute normalized to 1000 cells ( $\mathrm{pmol} \mathrm{O}_{2}$ per 1000 cells per min). The spare respiratory capacity (SRC) was calculated by subtracting the OCR values obtained after the injection of 2,4-dinitrophenol minus basal respiration values.

\section{Statistical analysis}

Statistical analyses were performed using STATISTICA software (Statsoft Inc., Tulsa, OK, USA). The data between different treatments were analysed using one-way ANOVA and Tukey's post hoc test; $P<0.05$ was considered as significant and $P<$ 0.01 highly significant. All the analyses were conducted in triplicate and data are reported as mean \pm SD.

\section{Results and discussion}

\section{Fruit composition and TAC}

The chemical composition and TAC of acerola fruit are shown in Table 1. The results show that acerola fruits are an important dietary source of several bioactive compounds with beneficial effects on health, such as polyphenols (16.40 mg GAE per $\mathrm{g}$ FW) and vitamin C (1201 mg per $100 \mathrm{~g}$ FW). ${ }^{22}$ In particular, the content of these beneficial compounds, measured in this study, is significantly higher than those previously reported in fruit such as strawberries, ${ }^{23}$ blackberries, ${ }^{24}$ raspberries $^{25}$ and cherries, ${ }^{5,26-28}$ which is in agreement with data previously reported by several authors. ${ }^{29,30}$ Moreover, for flavonoid content and anthocyanins, the values were within the range previously reported in other red fruit. ${ }^{23-25}$

Acerola fruits also represent an important natural source of folates, with contents of 5-methyltetrahydrofolic acid disodium salt (3.28 $\mu \mathrm{g}$ per $100 \mathrm{~g}$ FW), (6S)-5-formyl-5,6,7,8-tetrahydrofolic acid, calcium salt (79.82 $\mu \mathrm{g}$ per $100 \mathrm{~g} \mathrm{FW})$ and $(6 S)-5,6,7,8-$

Table 1 Chemical composition and total antioxidant capacity of acerola fruits

Values $^{a}$

\section{Parameters}

Total phenolic content (TPC) (mg GAE per $\mathrm{g}$ FW)

Total flavonoid content (TFC) (mg CatEq per g FW)

Total anthocyanin content (ACY) (mg PgEq per $\mathrm{g}$ FW)

Vitamin C content (VitC) (mg per $100 \mathrm{~g}$ FW)

$\beta$-Carotene content ( $\beta$ CarotC) $(\mu \mathrm{g}$ per $100 \mathrm{~g}$ FW)

$0.37 \pm 0.01$

$0.12 \pm 0.03$

$1201 \pm 72.11$

$32.11 \pm 4.31$

\section{Folate ( $\mu \mathrm{g}$ folate per $100 \mathrm{~g}$ FW)}

5-Methyltetrahydrofolic acid disodium salt

(6S)-5-Formyl-5,6,7,8-tetrahydrofolic acid, calcium salt (natural calcium folinate)

(6S)-5,6,7,8-Tetrahydrofolic acid

$3.28 \pm 0.02$

$79.82 \pm 3.21$

$12.62 \pm 0.16$

Total antioxidant capacity (TAC) ( $\mu$ mol TEq per $\mathrm{g}$ FW)

TEAC

DPPH

$132.64+12.26$

$8.41 \pm 2.18$

FRAP

$144.91 \pm 21.52$ 
tetrahydrofolic acid (12.62 $\mu \mathrm{g}$ per $100 \mathrm{~g}$ FW) higher than those previously reported in other red fruit such as strawberries. ${ }^{16,31}$ $\beta$-Carotene content was also analysed, showing that acerola fruit could be considered as an important source of this carotenoid in the diet, similar to other red fruit including strawberries, ${ }^{10}$ blackberries, ${ }^{24}$ raspberries ${ }^{25}$ and cherries. ${ }^{5,26-28}$

Anthocyanins, flavonols and the compositions of other phenolic derivatives in acerola fruit were determined by HPLC-DAD/ESI-MS ${ }^{n}$. In Fig. 1 representative HPLC chromatograms of the profiles obtained at $520 \mathrm{~nm}$, for anthocyanins (A), and other phenolics obtained at $330 \mathrm{~nm}$ (B) are shown. The compounds were identified on the basis of their UV and mass spectra obtained by HPLC-DAD-ESI/MS, as well as their chromatographic behaviour compared with external standards when available. UV spectra, mass characteristics and the identity of the peaks for anthocyanins are indicated in Table 2, while flavonols and other phenolic derivatives are shown in Table 3. Only two anthocyanins were found in acerola fruits (cyanidin 3-O-rhamnoside and pelargonidin 3-O-rhamnoside), while 18 peaks were assigned as flavonols and other phenolic derivatives. The principal phenolic acids identified were caffeoyl hexoside, dihydrocaffeoylquinic acid and coumaroyl hexoside, while the principal flavonoids were glycosylated forms of quercetin and kaempferol. These results are in agreement with previous reports, which indicated quercetin and kaempferol, ${ }^{32}$ and cyanidin and pelargonidin, and their glycosylated form, ${ }^{33}$ as the main flavonoids and anthocyanins present in acerola fruit, respectively.

The TAC of the hydroalcoholic extract was also determined using in parallel the TEAC, DPPH and FRAP assays, which highlighted the ability of the extract to scavenge $\mathrm{ABTS}^{{ }^{++}}$and $\mathrm{DPPH}^{\cdot}$ radicals and to reduce $\mathrm{Fe}^{3+}$ to $\mathrm{Fe}^{2+}$ (Table 1), demonstrating its antioxidant potential, in agreement with previous reports in other red fruit..$^{5,23-25,27}$

\section{Acerola treatment reduces the biomarkers of oxidative damage in HDFa}

Since the ability of several red fruit to protect against oxidative stress related diseases in humans is well documented, and
Table 2 Wavelengths of maximum absorption, mass spectral data and tentative identification of anthocyanin composition in acerola fruits

\begin{tabular}{lllll}
$\begin{array}{l}\text { Peak } \\
\text { number }\end{array}$ & $\lambda_{\max }(\mathrm{nm})$ & {$[\mathrm{M}]^{+}(\mathrm{m} / \mathrm{z})$} & $\mathrm{MS}^{2}$ & Identification \\
\hline A1 & 517 & 433 & 287 & Cyanidin 3-rhamnoside \\
A2 & 502 & 417 & 271 & Pelargonidin 3-rhamnoside
\end{tabular}

related to their antioxidant, anti-inflammatory, antihypertensive, anti-atherosclerotic and anti-cancer activities, ${ }^{3,23,24,34}$ the protective effects of an ACExt against oxidative damage using an in vitro HDFa model were analyzed. The preliminary cytotoxic assay showed no cytotoxic effect in HDFa after treatment with ACExt at the concentration tested (data not shown), while treatment with the oxidant AAPH caused a significant decrease in cellular vitality $(\sim 50 \%$ of vitality) $(P<0.05)$ compared with the control cells. Pre-treatment with ACExt was able to protect HDFa viability, showing a higher number of live cells $(P<0.05)$ compared with cells treated with AAPH. The protective capacity of ACExt toward cell viability was also studied in relation to the change in the number of live, dead and apoptotic cells after the different treatments. After ACExt treatment no significant changes were observed in apoptotic and dead cells compared with the control, while treatment with AAPH caused a significant increase $(P<0.05)$ in apoptotic and dead cells compared with the control. The protective effect of ACExt against cytotoxic damage mediated by oxidative stress was evident after pre-treatment with ACExt, which was able to significantly decrease $(P<0.05)$ the number of dead and apoptotic cells compared with cells under stress with AAPH (Fig. 2A). These results are in agreement with those previously reported by our group regarding the protective effect of a strawberry extract against oxidative damage in HDFa. That study reported an increasing cellular vitality in the cells pre-incubated with the strawberry extract and then treated with AAPH, showing a low number of apoptotic and dead cells compared with cells stressed with AAPH. ${ }^{10}$

The biological effects of red fruit have been largely attributed to their chemical components, among which is ACYs. In
A

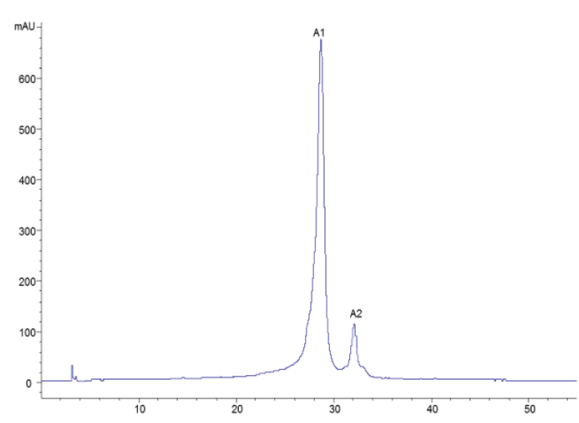

B

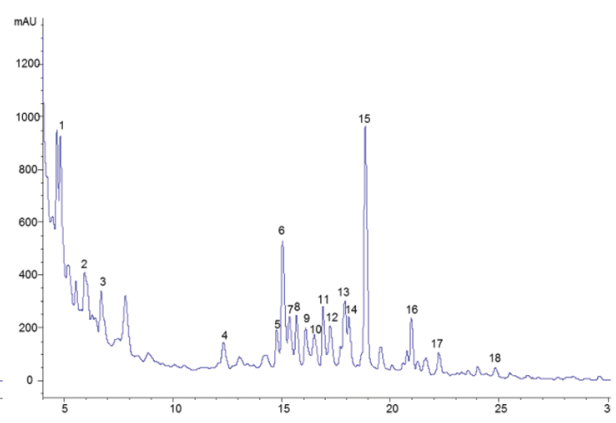

Fig. 1 HPLC profiles obtained at $520 \mathrm{~nm}$, for anthocyanin analysis (A), and for phenolic profiles obtained at $330 \mathrm{~nm}$ (B). Labelled peaks represent the compounds identified on the basis of their UV and mass spectra obtained by HPLC-DAD-ESI/MS, as well as their chromatographic behaviour compared to external standards and shown in Tables 2 and 3. 
Table 3 Wavelengths of maximum absorption, mass spectral data and tentative identification of flavonols and other phenolic derivatives in acerola fruits

\begin{tabular}{llllll}
\hline Peaks & $R_{\mathrm{t}}(\mathrm{min})$ & $\lambda_{\max }(\mathrm{nm})$ & Pseudomolecular ion $[\mathrm{M}-\mathrm{H}]^{-}(\mathrm{m} / \mathrm{z})$ & $\mathrm{MS}^{2}(\mathrm{~m} / \mathrm{z})$ & Tentative identification \\
\hline 1 & 4.8 & $285,300 \mathrm{sh}$ & 341 & - & 209,191 \\
2 & 5.9 & 315 & 355 & $163,145,119$ & Caffeoyl hexoside \\
3 & 6.7 & $283,306 \mathrm{sh}$ & 325 & Coumaroyl hexoside \\
4 & 12.3 & 256,353 & 741 & 301 & Quercetin $O$-hexose-deoxyhexose-pentoside \\
5 & 14.8 & 266,342 & 725 & 285,133 & Kaempferol $O$-hexose-deoxyhexose-pentoside \\
6 & 15.0 & 256,356 & 609 & 301 & Quercetin $O$-hexose-deoxyhexoside \\
7 & 15.4 & 254,355 & 609 & 301 & Quercetin $O$-hexose-deoxyhexoside \\
8 & 15.7 & 256,352 & 755 & 315,301 & Methylquercetin $O$-hexose-deoxyhexose-pentoside \\
9 & 16.1 & 255,355 & 463 & 301 & Quercetin $O$-hexoside \\
10 & 16.5 & 256,352 & 597 & 477,315 & Methylquercetin $O$-hexoside derivative \\
11 & 16.9 & 266,346 & 593 & 285 & Kaempferol $O$-hexose-deoxyhexoside \\
12 & 17.2 & 288 & 449 & 303,285 & Dihydroquercetin $O$-deoxyhexoside \\
13 & 17.9 & 265,350 & 593 & 285 & Kaempferol $O$-hexose-deoxyhexoside \\
14 & 18.1 & 259,349 & 447 & 285 & Kaempferol $O$-hexoside \\
15 & 18.8 & 255,354 & 447 & $301,283,271$ & Quercetin $O$-deoxyhexoside \\
16 & 20.9 & 261,349 & 431 & 285,255 & Kaempferol $O$-deoxyhexoside \\
17 & 22.3 & $270,300,352$ & 609 & 463,301 & Quercetin $O$-coumaroyl hexoside \\
18 & 24.8 & 360 & 301 & - & Quercetin
\end{tabular}

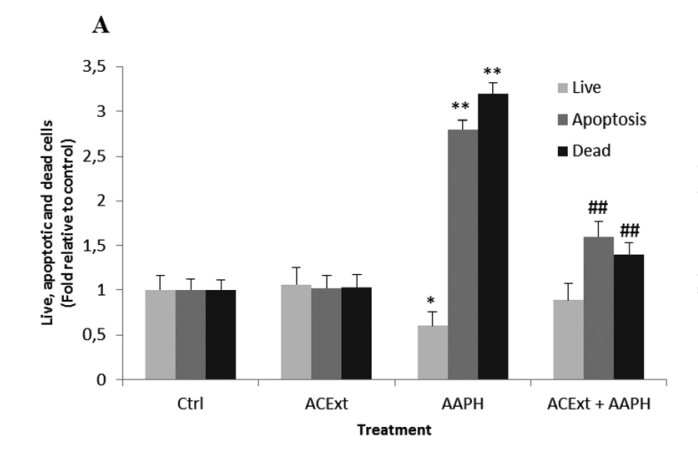

B

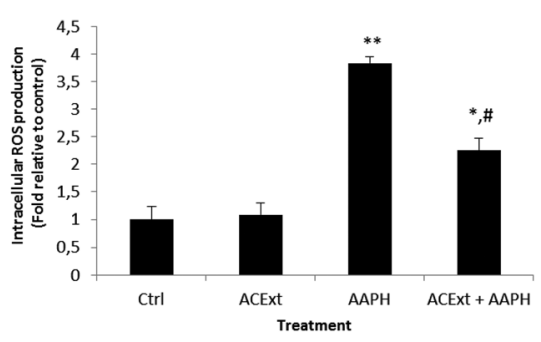

Fig. 2 (A) Percentage of live, dead and apoptotic cells after each treatment and (B) intracellular ROS generation in HDFa after each treatment. Cells were treated with (i) EMEM only (Ctrl), (ii) ACExt for $24 \mathrm{~h}$ (ACExt), (iii) AAPH (10 mM) for $24 \mathrm{~h}$ (AAPH) or (iv) ACExt for $24 \mathrm{~h}$ and then with AAPH $(10 \mathrm{mM})$ for $24 \mathrm{~h}(\mathrm{ACExt}+\mathrm{AAPH})$. Results are reported as mean \pm SD of three experiments. ${ }^{*} P<0.05, * * P<0.01$, significant differences compared to the control; ${ }^{\#} P<0.05,{ }^{\# \#} P<0.01$, significant differences between the AAPH and ACExt + AAPH groups.

fact, several studies demonstrated the capacity of ACYs to protect against apoptosis and oxidative damage in different cellular models. ${ }^{35-39}$ Studies about the polyphenol profile in acerola fruit have reported the presence of bioactive compounds, such as the ACYs cyanidin-3- $\alpha-O$-rhamnoside and pelargonidin-3- $\alpha$-O-rhamnoside, the flavonols quercetin-3-Orhamnoside and quercetin-3-O-galactoside, and proanthocyanidins, ${ }^{40,41}$ as well as other polyphenols like chlorogenic acid, (-)-epigallocatechin gallate, and (-)-epicatechin. ${ }^{42}$ All these compounds are closely related to beneficial biological properties, allowing the justification, at least in part, of the biological effects here observed.

The protective effect of ACExt against apoptosis could also be related to its effects on intracellular ROS levels and oxidative damage in lipids and proteins found in HDFa after the induction of oxidative stress. Intracellular ROS levels were significantly higher after incubation with AAPH compared with the control $(P<0.05)$, while pre-treatment with ACExt was able to significantly reduce $(P<0.05)$ those levels compared with the AAPH-stressed cells (Fig. 2B). Similar behaviour was observed in markers of oxidative damage to macromolecules such as lipids and proteins (Table 4). The markers of lipid oxidative damage (TBARS and hydroperoxides) and protein damage (protein carbonyl groups) significantly increased after incubation of HDFa with AAPH compared with the controls $(P<0.05)$, whereas pre-incubation with ACExt significantly reduced $(P<0.05)$ those markers compared with the AAPHstressed cells. The ACExt treatment was also able to improve the activity of the antioxidant enzymes SOD and CAT after oxidative damage. In the AAPH-stressed cells the activity of both enzymes was significantly affected compared with the control $(P<0.05)$, while after pre-treatment with ACExt the activity of these enzymes was significantly increased $(P<0.05)$ compared with the AAPH-stressed cells. These results are in line with previous reports that demonstrated the protective effects of polyphenols from different sources against the increase in intracellular ROS and oxidative damage using different cellular models exposed to different oxidative agents. ${ }^{5,10,15,16,34,43-46}$ More recently it has been demonstrated that the protective effects of polyphenols go beyond their simple antioxidant 
Table 4 Biomarkers of oxidative damage in HDFs

\begin{tabular}{|c|c|c|c|c|}
\hline Biomarkers of oxidative damage & Ctrl & ACExt & AAPH & ACExt + AAPH \\
\hline SOD activity (U per mg prot) & $19.21 \pm 1.20$ & $17.08 \pm 1.13$ & $4.75 \pm 1.32^{* *}$ & $11.18 \pm 1.41^{\#}$ \\
\hline Lipid hydroperoxides $(\mu \mathrm{M})$ & $41.70 \pm 3.54$ & $38.29 \pm 3.48$ & $72.35 \pm 2.41^{* *}$ & $47.35 \pm 4.19^{\# \#}$ \\
\hline Protein carbonyl (nmol per mg prot) & $0.63 \pm 0.12$ & $0.59 \pm 0.14$ & $2.10 \pm 0.11^{* *}$ & $1.20 \pm 0.12^{\# \#}$ \\
\hline
\end{tabular}

Results are reported as mean \pm SD of three experiments. ${ }^{*} P<0.05,{ }^{* *} P<0.01$, significant differences compared to the control $(\mathrm{Ctrl})$; ${ }^{\sharp} P<0.05$, ${ }^{\# \#} P<0.01$, significant differences between the AAPH and ACExt + AAPH groups.

effect, by activating certain molecular pathways related to the antioxidant response. ${ }^{3}$ Gasparrini et $a l^{16}$ and Lee et $a .^{34}$ reported that berry polyphenols were able to protect the LPSstimulated RAW 264.7 macrophages against oxidative damage through the activation of the nuclear factor-erythroid 2-related factor 2 (Nrf2). Similarly, Alvarez-Suarez et al. ${ }^{15}$ reported that polyphenols from Manuka honey could be responsible for their beneficial properties through the improvement of the antioxidant response in HDFa subjected to oxidative stress by the activation of the AMPK/ $p$-AMPK/Nrf2/ARE signalling pathway, supporting the potential effect of dietary polyphenols on protection against oxidative damage.

\section{Acerola treatment protects mitochondrial functionality against oxidative damage}

The electron transport chain in mitochondria is considered to be the most important intracellular source of ROS, ${ }^{45}$ hence the importance of ensuring the integrity of the mitochondrial function as a strategy to avoid the overproduction of intracellular ROS and their subsequent cellular damage. In this sense, the effect of ACExt against AAPH-induced stress on mitochon- drial functionality was investigated. After measuring basal OCR, cells were exposed sequentially to four modulators of oxidative phosphorylation (OXPHOS) (oligomycin, 2,4-DNP and antimycin/rotenone), and OCR was measured after the application of each of them. In AAPH-stressed cells basal OCR was markedly affected $(P<0.05)$ compared with the control cells, while pre-treatment with ACExt was able to protect mitochondria against oxidative damage showing OCR values that were significantly higher $(P<0.05)$ compared with the AAPHstressed cells (Fig. 3A). The spare respiratory capacity (SRC) was also determined as an indicator of the energy limit state of the cell after the different treatments. ${ }^{47}$ Similarly to the OCR, the SCR was significantly affected $(P<0.05)$ in the AAPHstressed cells, while ACExt pre-treatment resulted in a significant improvement $(P<0.05)$ compared with the AAPH-stressed cells (Fig. 3B). These results are in agreement with those previously described by Giampieri et al., ${ }^{10}$ who reported that an anthocyanin-rich strawberry extract was able to protect the mitochondria in HDF against the oxidative damage mediated by AAPH, while Alvarez-Suarez et al. ${ }^{15}$ reported that pre-incubation of HDF with Manuka honey was also able to protect
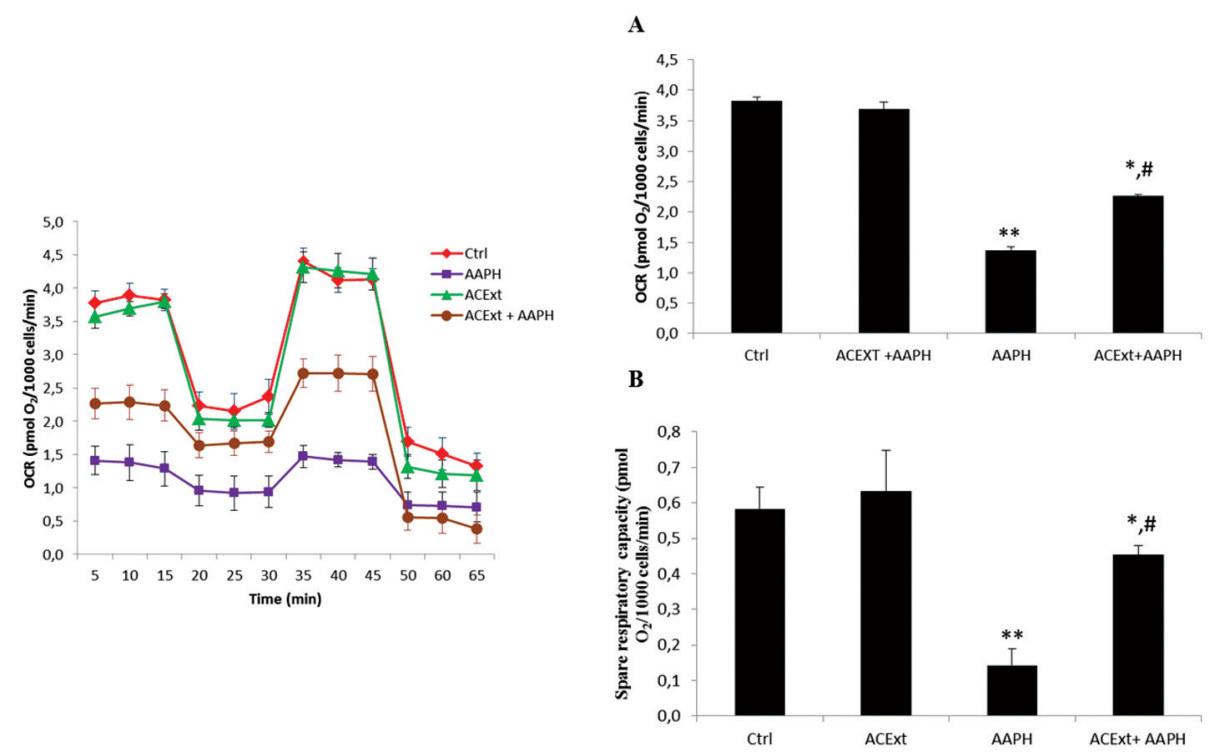

Fig. 3 Oxygen consumption rate (OCR). OCR was monitored using the Seahorse XF-24 Extracellular Flux Analyzer with the sequential injection of oligomycin, 2,4-DNP, and rotenone/antimycin. (A) Basal OCR levels and (B) spare respiratory capacity in cells. Results are reported as mean \pm SD of three experiments. ${ }^{*} P<0.05$, ${ }^{*} P<0.01$, significant differences compared to the control; ${ }^{\#} P<0.01$, ${ }^{\# \#} P<0.01$, significant differences between the $\mathrm{AAPH}$ and ACExt + AAPH groups. 
mitochondrial function against the damage produced by the AAPH oxidant. Similarly, Gasparrini et al. ${ }^{16}$ also informed that pre-treatment with a crude extract of strawberry polyphenols was able to protect the mitochondrial function in RAW macrophages against LPS-induced damage through the activation of the Nrf2 pathway, which is markedly AMPK-dependent. More recently it was also demonstrated that anthocyanin-rich strawberry consumption ameliorates age-associated impairments and mitochondrial biogenesis and functionality through the AMP-activated protein kinase-signalling cascade. ${ }^{48}$ All these results may support the hypothesis that the protective effect of polyphenols against oxidative stress could be related to their ability to up-regulate the AMPK/Nrf2/ARE (Kelch ECH associating protein 1/NF-E2-related factor 2/antioxidant responsive elements) signalling pathway, and the expression of antioxidant enzymes such as SOD and CAT, ${ }^{49}$ protecting macromolecules, cytoplasmic organelles and cells in general from oxidative damage. ${ }^{15,49}$

According to the results presented here, acerola fruits can be proposed as an important natural source of bioactive compounds with beneficial properties for health, such as polyphenols, vitamin $\mathrm{C}$ and folates. These results reinforce the perception of acerola as a fruit with great health benefits, showing that its biological properties are fundamentally based on its chemical composition, antioxidant capacity and ability to protect macromolecules against oxidative damage, through the stimulation of the antioxidant response, e.g., increase in antioxidant enzyme activity, and the protection of mitochondrial functionality.

\section{Conflicts of interest}

The authors declare no conflicts of interest.

\section{References}

1 D. Del Rio, A. Rodriguez-Mateos, J. P. E. Spencer, M. Tognolini, G. Borges and A. Crozier, Antioxid. Redox Signaling, 2013, 18, 1818-1892.

2 A. Bach-Faig, E. M. Berry, D. Lairon, J. Reguant, A. Trichopoulou, S. Dernini, F. X. Medina, M. Battino, R. Belahsen, G. Miranda and L. Serra-Majem, Public Health Nutr., 2011, 14, 2274-2284.

3 F. Giampieri, J. M. Alvarez-Suarez and M. Battino, J. Agric. Food Chem., 2014, 62, 3867-3876.

4 P. D. Johnson, World Rev. Nutr. Diet., 2003, 91, 67-75.

5 J. M. Alvarez-Suarez, E. Carrillo-Perdomo, A. Aller, F. Giampieri, M. Gasparrini, L. González-Pérez, P. BeltránAyala and M. Battino, Food Chem. Toxicol., 2017, 102, 4652.

6 K. Slinkard and V. L. Singleton, Am. J. Enol. Vitic., 1977, 28, 49-55.

7 V. Dewanto, X. Wu, K. K. Adom and R. H. Liu, J. Agric. Food Chem., 2002, 50, 3010-3014.
8 M. M. Giusti and R. E. Wrolstad, in Current Protocols in Food Analytical Chemistry, John Wiley \& Sons, Inc., 2001, DOI: 10.1002/0471142913.faf0102s00.

9 S. Tulipani, B. Mezzetti, F. Capocasa, S. Bompadre, J. Beekwilder, C. H. R. de Vos, E. Capanoglu, A. Bovy and M. Battino, J. Agric. Food Chem., 2008, 56, 696-704.

10 F. Giampieri, J. M. Alvarez-Suarez, L. Mazzoni, T. Y. ForbesHernandez, M. Gasparrini, A. M. Gonzalez-Paramas, C. Santos-Buelga, J. L. Quiles, S. Bompadre, B. Mezzetti and M. Battino, Food Funct., 2014, 5, 1939-1948.

11 M. J. I. Shohag, Y. Y. Wei, N. Yu, J. Zhang, K. Wang, J. Patring, Z. L. He and X. E. Yang, J. Agric. Food Chem., 2011, 59, 12520-12526.

12 R. Re, N. Pellegrini, A. Proteggente, A. Pannala, M. Yang and C. Rice-Evans, Free Radical Biol. Med., 1999, 26, 12311237.

13 I. F. F. Benzie and J. J. Strain, Anal. Biochem., 1996, 239, 7076.

14 A. Prymont-Przyminska, A. Zwolinska, A. Sarniak, A. Wlodarczyk, M. Krol, M. Nowak, J. de Graft-Johnson, G. Padula, P. Bialasiewicz, J. Markowski, K. P. Rutkowski and D. Nowak, J. Clin. Biochem. Nutr., 2014, 55, 48-55.

15 J. M. Alvarez-Suarez, F. Giampieri, M. Cordero, M. Gasparrini, T. Y. Forbes-Hernández, L. Mazzoni, S. Afrin, P. Beltrán-Ayala, A. M. González-Paramás, C. Santos-Buelga, A. Varela-Lopez, J. L. Quiles and M. Battino, J. Funct. Foods, 2016, 25, 38-49.

16 M. Gasparrini, T. Y. Forbes-Hernandez, F. Giampieri, S. Afrin, J. M. Alvarez-Suarez, L. Mazzoni, B. Mezzetti, J. L. Quiles and M. Battino, Food Chem. Toxicol., 2017, 102, 1-10.

17 H. Aebi, in Methods in Enzymology, Academic Press, 1984, vol. 105, pp. 121-126.

18 P. Kakkar, B. Das and P. N. Viswanathan, Indian J. Biochem. Biophys., 1984, 21, 130-132.

19 R. L. Levine, D. Garland, C. N. Oliver, A. Amici, I. Climent, A.-G. Lenz, B.-W. Ahn, S. Shaltiel and E. R. Stadtman, in Methods in Enzymology, Academic Press, 1990, vol. 186, pp. 464-478.

20 H. Ohkawa, N. Ohishi and K. Yagi, Anal. Biochem., 1979, 95, 351-358.

21 Z.-Y. Jiang, J. V. Hunt and S. P. Wolff, Anal. Biochem., 1992, 202, 384-389.

22 P. Gomez, M. Reynes, M. Dornier and J. P. Hebert, Fruits, 1999, 54, 247-260.

23 F. Giampieri, S. Tulipani, J. M. Alvarez-Suarez, J. L. Quiles, B. Mezzetti and M. Battino, Nutrition, 2012, 28, 9-19.

24 L. Kaume, L. R. Howard and L. Devareddy, J. Agric. Food Chem., 2012, 60, 5716-5727.

25 A. V. Rao and D. M. Snyder, J. Agric. Food Chem., 2010, 58, 3871-3883.

26 G. Ballistreri, A. Continella, A. Gentile, M. Amenta, S. Fabroni and P. Rapisarda, Food Chem., 2013, 140, 630638.

27 A. Nawirska-Olszańska, J. Kolniak-Ostek, M. Oziembłowski, A. Ticha, R. Hyšpler, Z. Zadak, P. Židová and F. Paprstein, Food Chem., 2017, 228, 136-142. 
28 J. Cao, Q. Jiang, J. Lin, X. Li, C. Sun and K. Chen, Food Chem., 2015, 173, 855-863.

29 M. Paz, P. Gúllon, M. F. Barroso, A. P. Carvalho, V. F. Domingues, A. M. Gomes, H. Becker, E. Longhinotti and C. Delerue-Matos, Food Chem., 2014, 172, 462-468.

30 D. Rinaldo, B. Fils-Lycaon and D. Mbéguié-A-Mbéguié, in Acta Horticulturae, 2014, vol. 1040, pp. 261-268.

31 S. Tulipani, S. Romandini, J. M. A. Suarez, F. Capocasa, B. Mezzetti, F. Busco, F. Bamonti, C. Novembrino and M. Battino, BioFactors, 2008, 34, 47-55.

32 G. A. Bataglion, F. M. A. da Silva, M. N. Eberlin and H. H. F. Koolen, Food Chem., 2015, 180, 280-287.

33 V. Vera de Rosso, S. Hillebrand, E. Cuevas Montilla, F. O. Bobbio, P. Winterhalter and A. Z. Mercadante, J. Food Compos. Anal., 2008, 21, 291-299.

34 S. G. Lee, B. Kim, Y. Yang, T. X. Pham, Y.-K. Park, J. Manatou, S. I. Koo, O. K. Chun and J.-Y. Lee, J. Nutr. Biochem., 2014, 25, 404-411.

35 J. Paixão, T. C. P. Dinis and L. M. Almeida, Apoptosis, 2011, 16, 976.

36 S. ali Shah, I. Ullah, H. Y. Lee and M. O. Kim, Mol. Neurobiol., 2013, 48, 257-269.

37 Y. Hu, Y. Ma, S. Wu, T. Chen, Y. He, J. Sun, R. Jiao, X. Jiang, Y. Huang, L. Deng and W. Bai, Front. Pharmacol., 2016, 7, 301.

38 J. M. Silvan, M. Reguero and S. de Pascual-Teresa, Food Funct., 2016, 7, 1067-1076.
39 H. B. Park, Y. S. Hah, J. W. Yang, J. B. Nam, S. H. Cho and S. T. Jeong, J. Orthop. Res., 2010, 28, 1162-1169.

40 T. Hanamura and H. Aoki, J. Food Sci., 2008, 73, T55-T61.

41 T. Hanamura, T. Hagiwara and H. Kawagishi, Biosci., Biotechnol., Biochem., 2005, 69, 280-286.

42 T. Mezadri, D. Villaño, M. S. Fernández-Pachón, M. C. García-Parrilla and A. M. Troncoso, J. Food Compos. Anal., 2008, 21, 282-290.

43 H. Zhang and R. Tsao, Curr. Opin. Food Sci., 2016, 8, 33-42.

44 S. Hooshmand, A. Kumar, J. Y. Zhang, S. A. Johnson, S. C. Chai and B. H. Arjmandi, Food Funct., 2015, 6, 17191725.

45 T. Y. Forbes-Hernández, F. Giampieri, M. Gasparrini, L. Mazzoni, J. L. Quiles, J. M. Alvarez-Suarez and M. Battino, Food Chem. Toxicol., 2014, 68, 154-182.

46 F. Jiménez-Aspee, C. Theoduloz, F. Ávila, S. Thomas-Valdés, C. Mardones, D. Von Baer and G. Schmeda-Hirschmann, Food Chem., 2016, 194, 908-919.

47 M. D. Brand and D. G. Nicholls, Biochem. J., 2011, 435, 297-312.

48 F. Giampieri, J. M. Alvarez-Suarez, M. D. Cordero, M. Gasparrini, T. Y. Forbes-Hernandez, S. Afrin, C. SantosBuelga, A. M. González-Paramás, P. Astolfi, C. Rubini, A. Zizzi, S. Tulipani, J. L. Quiles, B. Mezzetti and M. Battino, Food Chem., 2017, 234, 464-471.

49 I. Buendia, P. Michalska, E. Navarro, I. Gameiro, J. Egea and R. León, Pharmacol. Ther., 2016, 157, 84-104. 